Arbitrada Interdisciplinaria de Ciencias de la Salud. SALUD Y VIDA

Volumen 3. Número 6. Año 3. Julio - Diciembre 2019

Hecho el depósito de Ley: FA2016000010

ISSN: $2610-8038$

FUNDACIÓN KOINONIA (F.K)

Santa Ana de Coro, Venezuela.

Andrés Cruz Medina; Santiago Martínez Oñate

http://dx.doi.org/10.35381/s.v.v3i6.435

\title{
Conocimiento y práctica en el autocuidado de pacientes hipertensos
}

Knowledge and practice in self-care of hypertensive patients

\author{
Andrés Cruz Medina \\ andy1ppb92@gmail.com \\ Investigador Independiente \\ Ecuador \\ https://orcid.org/0000-0003-2971-1984 \\ Santiago Martínez Oñate \\ santyppb@gmail.com \\ Investigador Independiente \\ Ecuador \\ https://orcid.org/0000-0002-4164-7324
}

Recibido: 1 de mayo de 2019

Aprobado: 1 de junio de 2019

\section{RESUMEN}

La investigación tuvo como objetivo determinar la relación entre el conocimiento y práctica en el autocuidado de pacientes hipertensos de la provincia de Manabí - Ecuador. se generó un tipo de investigación correlacional transeccional. La población de estudio estuvo conformada por 138 pacientes hipertensos que acuden regularmente a los centros asistenciales de la provincia de Manabí. Se aplicó un programa formativo en conocimiento y práctica en el autocuidado de pacientes hipertensos de la provincia de Manabí Ecuador, con la finalidad de generar una cultura asociada al autocuidado y prevención de enfermedades. La correlación al ser -,331 significativa en el nivel 0,01 (bilateral), implica que existe relación entre ambas variables de forma proporcionar. Al aceptarse la hipótesis alterna, la relación de las variables permite concluir que los pacientes se encuentran en un estado de conciencia que les permite asumir con responsabilidad su autocuidado en función de la hipertensión arterial.

Descriptores: Política de salud; Epidemiología; Medicina; Preventiva; Lucha contra las enfermedades; Educación sanitaria. 


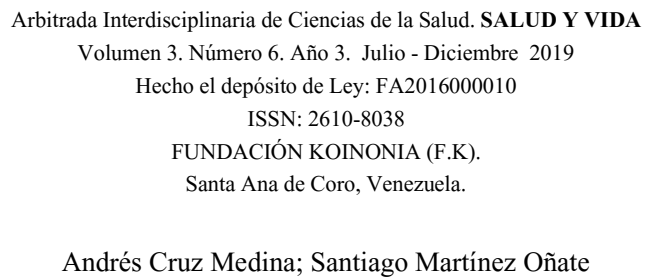

Andrés Cruz Medina; Santiago Martínez Oñate

\begin{abstract}
The research aimed to determine the relationship between knowledge and practice in the self-care of hypertensive patients in the province of Manabí - Ecuador. A type of translational correlational research was generated. The study population consisted of 138 hypertensive patients who regularly attend the health centers in the province of Manabí. A training program in knowledge and practice was applied in the self-care of hypertensive patients in the province of Manabí - Ecuador, in order to generate a culture associated with self-care and disease prevention. The correlation being -, 331 significant at the 0.01 level (bilateral), implies that there is a relationship between both variables in a proportionate way. When the alternative hypothesis is accepted, the relationship of the variables allows us to conclude that the patients are in a state of consciousness that allows them to assume their self-care responsibly according to arterial hypertension.
\end{abstract}

Descriptors: Health policy; Epidemiology; Preventive medicine; Disease control; Health education.

\title{
INTRODUCCIÓN
}

El conocimiento y práctica en el autocuidado de pacientes hipertensos, es una modalidad de importancia para el cuidado de la salud y la preservación de la vida, por cuanto el paciente adopta una serie de recomendaciones médicas para mantener niveles óptimo de tensión arterial con la finalidad de vivir con calidad, evitándose efectos secundarios que podrían conducir a la muerte. (Diario El Comercio , 2016), señala que:

En Ecuador, según datos de la Organización Mundial de la Salud (OMS), estas son la primera causa de muerte. Las enfermedades cardíacas isquémicas se encuentran en el primer puesto con un porcentaje total de $10,3 \%$ de las muertes registradas en el año 2012. Por otro lado, los paros cardíacos (también relacionados a problemas cardiovasculares) corresponden al 7,7\% del total de muertes. Según datos recogidos por el Instituto Nacional de Estadísticas y Censos del Ecuador (INEC), en el año 2014 se reportaron un total de 4430 muertes por enfermedades isquémicas del corazón, mientras que por insuficiencia cardíaca, los fallecimientos llegaron a 1316 . Las arritmias cardíacas sumaron un total de 168 muertes, mientras que los fallecidos por paros cardíacos en ese mismo año fueron 106. Del total de muertes por enfermedades del corazón, que suman casi 12 000 , el $51,68 \%$ de las víctimas son hombres, mientras que el $48,32 \%$ restantes son mujeres, según destacan las cifras recogidas por el INEC. 


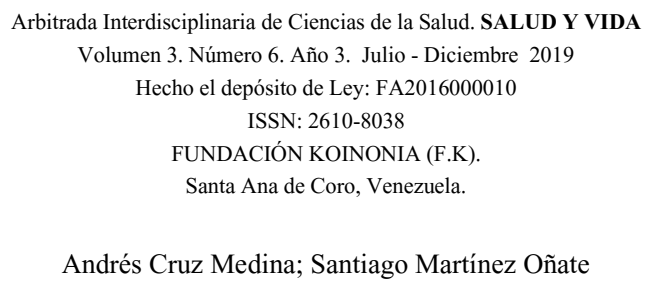

Andrés Cruz Medina; Santiago Martínez Oñate

El problema de hipertensión en el Ecuador, presenta cifras alarmantes, lo cual indica que las personas conciben un estilo de vida favorecedor de esta enfermedad e incluso el agravamiento de la misma, siendo necesario generar mecanismos preventivos en proporción de formar a la población a contraer un hábito de vida saludable como primer factor preventivo, luego promover el cuidado según las recomendaciones médicas para quienes sufren de hipertensión arterial. (Veletanga, 2019), reitera que la principal muerte en el Ecuador, se produce a causa de enfermedades cardiovasculares, promoviendo que un diagnóstico a tiempo, puede prevenir la muerte y la enfermedad. (Coello, 2017), alude que recién en el Ecuador se interesan por el cuidado de las enfermedades cardiovasculares.

De ese modo, el tema del autocuidado de la hipertensión arterial cobra vigencia, siendo necesaria implementar acciones que contribuyan a fortalecer la cultura de prevención en la población, (Organización Mundial de la Salud , 2018), describe:

La hipertensión, también conocida como tensión arterial alta o elevada, es un trastorno en el que los vasos sanguíneos tienen una tensión persistentemente alta, lo que puede dañarlos. Cada vez que el corazón late, bombea sangre a los vasos, que llevan la sangre a todas las partes del cuerpo. La tensión arterial es la fuerza que ejerce la sangre contra las paredes de los vasos (arterias) al ser bombeada por el corazón. Cuanta más alta es la tensión, más esfuerzo tiene que realizar el corazón para bombear. La mayoría de las personas con hipertensión no muestra ningún síntoma. En ocasiones, la hipertensión causa síntomas como dolor de cabeza, dificultad respiratoria, vértigos, dolor torácico, palpitaciones del corazón y hemorragias nasales, pero no siempre. Si no se controla, la hipertensión puede provocar un infarto de miocardio, un ensanchamiento del corazón y, a la larga, una insuficiencia cardiaca (p. 1).

Es necesario evitar problemas cardiovasculares derivados de la hipertensión arterial, de ese modo, se genera la posibilidad de contar con una población saludable que pueda generar aportes al Estado en función de ser productivo, minimizando la inversión pública en atención hospitalaria (Angarita, 2014), plantea un importante;

Reto para enfermería en la promoción de la salud con la estructuración de propuestas educativas para el autocuidado que motiven a los hipertensos en la decisión de cambio, a la modificación de su comportamiento, en la 
Arbitrada Interdisciplinaria de Ciencias de la Salud. SALUD Y VIDA

Volumen 3. Número 6. Año 3. Julio - Diciembre 2019

Hecho el depósito de Ley: FA2016000010

ISSN: $2610-8038$

FUNDACIÓN KOINONIA (F.K)

Santa Ana de Coro, Venezuela.

Andrés Cruz Medina; Santiago Martínez Oñate

adopción de conductas sobre estilos de vida saludables y en el fortalecimiento de su actitud para comprometerse en el cuidado de sí mismo (p. 143).

Las agencia de autocuidado proporcionan educación en función de las necesidades del paciente, de ese modo, se contribuye en generar un estilo de vida saludable en función de que el paciente adopte el autocuidado como medida principal para la prevención y control de hipertensión arterial, siendo necesario configurar una aptitud basada en la búsqueda de la salud como motor principal de calidad de vida. Para tal fin se toma como referencia la teoría del autocuidado de Dorothea Orem, (Acosta, 2011), explica que:

El autocuidado es una función humana reguladora que debe aplicar cada individuo de forma deliberada con el fin de mantener su vida y su estado de salud, desarrollo y bienestar, por tanto es un sistema de acción. La elaboración de los conceptos de autocuidado, necesidad de autocuidado, y actividad de autocuidado conforman los fundamentos que permiten entender las necesidades y las limitaciones de acción de las personas que pueden beneficiarse de la enfermería. Como función reguladora del hombre, el autocuidado es diferente de otros tipos de regulación del funcionamiento y el desarrollo humano.

Así la teoría del autocuidado, se complementa con la teoría del déficit del autocuidado y teoría de sistema de enfermería, siendo este el principal aporte de Dorothea Orem, con la finalidad establecer una escala conocida como "Escala de Apreciación de Agencia de Autocuidado (ASA)", la cual permite al paciente auto administrar valoración sobre la prevención y manejo de enfermedades, siendo en la actual investigación, aplicada en pacientes con hipertensión arterial. 
Arbitrada Interdisciplinaria de Ciencias de la Salud. SALUD Y VIDA

Volumen 3. Número 6. Año 3. Julio - Diciembre 2019

Hecho el depósito de Ley: FA2016000010

ISSN: $2610-8038$

FUNDACIÓN KOINONIA (F.K)

Santa Ana de Coro, Venezuela.

Andrés Cruz Medina; Santiago Martínez Oñate

Escala de Apreciación de Agencia de Autocuidado (ASA).

Figura 1.

Ítem

ASA01. A medida que cambian las circunstancias voy haciendo ajustes para mantenerme con salud

ASA 02. Reviso si son buenas las formas que practico habitualmente para mantenerme con salud

ASA03. Si tengo problemas para moverme o desplazarme me las arreglo para conseguir ayuda

ASA04. Puedo hacer lo necesario para mantener limpio el ambiente donde vivo

ASA05. Hago en primer lugar lo que sea necesario para mantenerme con salud

ASA06. Me faltan las fuerzas necesarias para cuidarme como debo

ASA07. Puedo buscar mejores formas para cuidar mi salud que las que tengo ahora

ASA08. Cambio la frecuencia con que me baño para mantenerme limpio

ASA09. Para mantener el peso que me corresponde hago cambios en mis hábitos alimenticios

ASA10. Cuando hay situaciones que me afectan, las manejo de manera que pueda mantener mi forma de ser

ASA11. Pienso en hacer ejercicio y descansar un poco durante el dia, pero no llego a hacerlo

ASA12. Cuando necesito ayuda puedo recurrir a mis amigos de siempre

ASA13. Puedo dormir lo suficiente como para sentirme descansado

ASA14. Cuando tengo información sobre mi salud pido explicaciones sobre lo que no entiendo

ASA15. Examino mi cuerpo para ver si hay algún cambio

ASA16. He sido capaz de cambiar hábitos que tenía muy arraigados con tal de mejorar mi salud

ASA17. Cuando tengo que tomar una nueva medicina cuento con

una persona que me brinda información sobre los efectos secundarios

ASA18. Soy capaz de tomar medidas para garantizar que mi familia y yo no corramos peligro

ASA19. Soy capaz de evaluar qué tanto me sirve lo que hago para mantenerme con salud

ASA 20. Debido a mis ocupaciones diarias me resulta difícil sacar tiempo para cuidarme

ASA21. Si mí salud se ve afectada yo puedo conseguir la información necesaria sobre qué hacer

ASA22. Si no puedo cuidarme puedo buscar ayuda

ASA23. Puedo sacar tiempo para mi

ASA24. A pesar de las limitaciones para movilizarme, soy capaz de

cuidarme como a mi me gusta

Tomado de Rodríguez Gázquez, Ma de los Ángeles, Arredondo Holguín, Edith, \& Salamanca Acevedo, Yurany Andrea. (2013).

La figura 1, representa la escala ASA, adaptada a la realidad colombiana, por lo tanto se toma por similitud demográfica para ser aplicada en la población ecuatoriana objeto de estudio de la presente investigación, de ese modo, se trabajó con instrumentos contextualizados a la realidad social del Ecuador. Prosiguiendo con lo planteado, Backes, 
Erdmann, Büscher (2010), señalan los siguientes aspectos relacionados a la teoría del autocuidado:

1. El cuidar de uno mismo: es el proceso de autocuidado se inicia con la toma de conciencia del propio estado de salud. Esta toma de conciencia requiere en si misma el pensamiento racional para aprovechar la experiencia personal, normas culturales y de conductas aprendidas, a fin de tomar una decisión sobre el estado de salud, en el cual debe de existir el deseo y el compromiso de continuar con el plan.

2. Limitaciones del cuidado: son las barreras o limitaciones para el autocuidado que son posible, cuando la persona carece de conocimientos suficientes sobre sí misma y quizás no tenga deseos de adquirirlos. Puede presentar dificultades para emitir juicios sobre su salud y saber cuándo es el momento de buscar ayuda y consejo de otros.

3. El aprendizaje del autocuidado: el aprendizaje del autocuidado y de su mantenimiento continuo son funciones humanas. Los requisitos centrales para el autocuidado con el aprendizaje y el uso del conocimiento para la realización de secuencias de acciones de autocuidado orientadas internas y externamente. Si una acción de autocuidado es interna o externa en su orientación puede determinarse mediante la observación, obteniendo datos subjetivos del agente de autocuidado o de ambas manera

El autocuidado requiere que el paciente tenga motivación y disciplina para asumir con eficacia el proceso de autocuidado, de ese modo, generará hábito de vida saludable que le permita controlar, mejorar, su condición médica, siendo necesaria el asesoramiento en los primeros días o meses para una adecuada adaptación, situación que permitirá generar mayores posibilidades de que el paciente obtenga cambios significativos en su salud. 
Arbitrada Interdisciplinaria de Ciencias de la Salud. SALUD Y VIDA

Volumen 3. Número 6. Año 3. Julio - Diciembre 2019

Hecho el depósito de Ley: FA2016000010

ISSN: 2610-8038

FUNDACIÓN KOINONIA (F.K)

Santa Ana de Coro, Venezuela.

Andrés Cruz Medina; Santiago Martínez Oñate

Figura 2. Teorías de Orem.

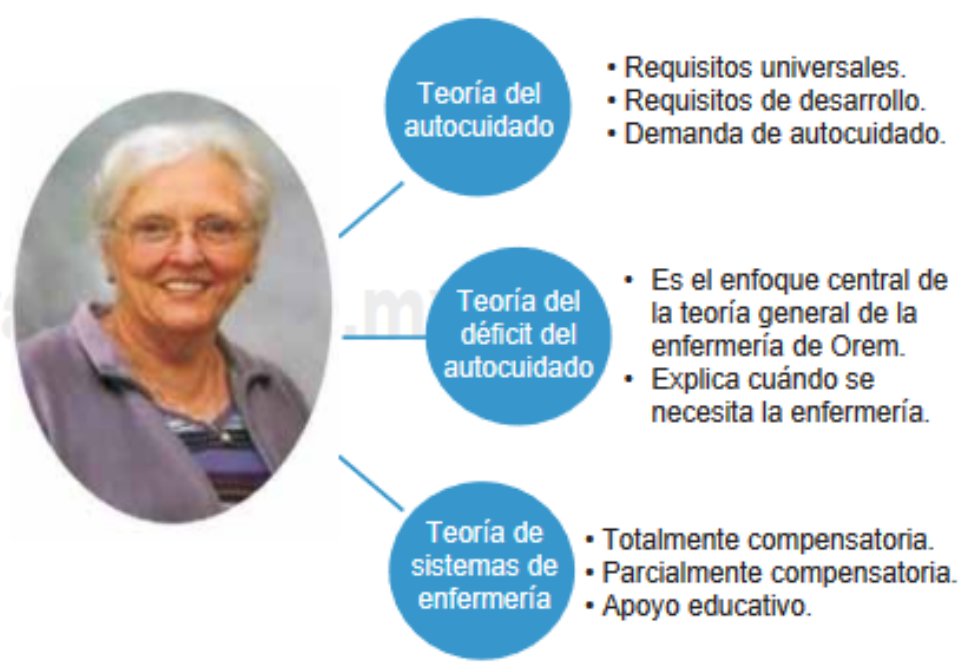

Tomado de Margarita Pereda Acosta (2011).

Las teorías de Orem, permiten configurar acciones para el autocuidado de pacientes en apoyo de las profesionales de la enfermería, así se concibe la salud como un proceso donde interviene médico - enfermera - paciente, con la finalidad de generar un estilo de prevención y control sanitaria que permita elevar la calidad de vida de las personas en concordancia con las políticas del buen vivir. En este sentido, la actual investigación tiene como objetivo determinar la relación entre el conocimiento y práctica en el autocuidado de pacientes hipertensos de la provincia de Manabí, Ecuador con la finalidad de propiciar la formación de los pacientes en la adopción del enfoque del autocuidado como generador de un proceso preventivo de salud, por cuanto al ser diagnosticada la hipertensión arterial, esta se puede controlar para evitar riesgos mayores como enfermedades cardiovasculares e incluso la muerte por infarto al miocardio.

\section{METODOLOGÍA DE LA INVESTIGACIÓN}

Se aplicó un programa formativo en conocimiento y práctica en el autocuidado de pacientes hipertensos de la provincia de Manabí - Ecuador, con la finalidad de generar una cultura asociada al autocuidado y prevención de enfermedades, el programa se generó en sesiones basadas en conocimiento sobre las teorías del autocuidado, manejo 
de la escala ASA, riesgos de la hipertensión arterial, factores de prevención como alimentación, deportes, vida saludable, asistencia a control médico y valoración de la escala ASA, durante el segundo semestre 2018.

Basado en lo planteado, al final se generó un tipo de investigación correlacional transeccional, lo cual permitió conocer la relación del conocimiento y práctica en el autocuidado en pacientes hipertensos, para lo cual se sustentó en Hernández, Fernández, Baptista (2014). La población de estudio estuvo conformada por 138 pacientes hipertensos que acuden regularmente a los centros asistenciales de la provincia de Manabí.

\section{RESULTADOS}

Correlación del conocimiento y práctica en el autocuidado en pacientes hipertensos. Cuadro 1.

\begin{tabular}{llr|r} 
& & Autocuidado & Hipertensos \\
\hline Autocuidado & Correlación de Pearson & 1 &,$- 331^{\star \star}$ \\
\cline { 2 - 4 } & Sig. (bilateral) & &, 000 \\
\cline { 2 - 4 } & $\mathrm{N}$ & 138 & 138 \\
\hline Hipertensos & Correlación de Pearson &,$- 331^{\star \star}$ & 1 \\
\cline { 2 - 4 } & Sig. (bilateral) &, 000 & \\
\cline { 2 - 4 } & $\mathrm{N}$ & 138 & 138 \\
\hline
\end{tabular}

**. La correlación es significativa en el nivel 0,01 (bilateral).

La correlación al ser -,331 significativa en el nivel 0,01 (bilateral), implica que existe relación entre ambas variables de forma proporcionar, es así que se responden a las siguientes hipótesis:

Hipótesis Nula H0: No existe relación entre el conocimiento y práctica en el autocuidado en pacientes hipertensos. 
Arbitrada Interdisciplinaria de Ciencias de la Salud. SALUD Y VIDA

Volumen 3. Número 6. Año 3. Julio - Diciembre 2019

Hecho el depósito de Ley: FA2016000010

ISSN: $2610-8038$

FUNDACIÓN KOINONIA (F.K).

Santa Ana de Coro, Venezuela.

Andrés Cruz Medina; Santiago Martínez Oñate

Hipótesis Alterna H1: Existe relación entre el conocimiento y práctica en el autocuidado en pacientes hipertensos.

Por consiguiente se acepta la Hipótesis Alterna H1: Existe relación entre el conocimiento y práctica en el autocuidado en pacientes hipertensos.

\section{DISCUSIÓN}

Los pacientes objeto de estudio han adoptado de modo favorable el conocimiento del autocuidado como factor de cuidado, aunado que la escala ASA, permite generar una conducta preventiva no solo en relación a la hipertensión, sino que indirectamente favorece la salud de modo integral, de ese modo, se concuerda con Naranjo Hernández, Concepción Pacheco, \& Rodríguez Larreynaga, (2017), quienes indican que:

El logro de la toma de conciencia del autocuidado que requiere la persona afectada con alguna enfermedad en función de mejorar o mantener el estado de salud, a través de las posibilidades que le brinda el personal de Enfermería debe nutrirse de las experiencias personales, normas culturales y conductas aprendidas, modificables o no, crear así los niveles de compromiso, para conservar la salud y bienestar de las personas. Las formas de llevar a cabo el autocuidado son propias de cada persona y están influidos por los valores culturales del grupo social al que pertenece que condicionan su realización y la forma de hacerlo requiere para ello de conocimientos.

El autocuidado requiere aceptación y adopción por parte del paciente, siendo este el principal protagonista en el proceso, de él depende la efectividad de los programas que se puedan implementar para la prevención y manejo asertivo de enfermedades a partir del autocuidado, siendo necesario fomentar el conocimiento en este sentido para que los pacientes puedan formar una conciencia acorde en razón de cumplir las metas propuestas al iniciar el autocuidado.

\section{CONCLUSIONES}

El trabajo de investigación presentado se muestra como un enfoque de prevención integral de la salud, Aldana Zavala \& Isea Argüelles (2019), sostienen la visión de la 
Arbitrada Interdisciplinaria de Ciencias de la Salud. SALUD Y VIDA

Volumen 3. Número 6. Año 3. Julio - Diciembre 2019

Hecho el depósito de Ley: FA2016000010

ISSN: $2610-8038$

FUNDACIÓN KOINONIA (F.K)

Santa Ana de Coro, Venezuela.

Andrés Cruz Medina; Santiago Martínez Oñate

epidemiología social permite trabajar desde una concepción de atención preventiva del ser humano, teniendo como escenario el contexto cultural, social, laboral, educativo, donde se interrelaciona con el medio ambiente, de ese modo, se transciende el paradigma de atención reactiva al de atención preventiva en salud, siendo esto concomitante con el autocuidado como método de generación en el paciente, la posibilidad de asumir con responsabilidad su cuidado a partir de las prescripciones médicas.

Al aceptarse la hipótesis alterna, la relación de las variables permite concluir que los pacientes se encuentran en un estado de conciencia que les permite asumir con responsabilidad su autocuidado en función de la hipertensión arterial, de ese modo, se cuenta con personas enfocadas a su cuidado, lo cual implica estar en concordancia con las políticas del buen vivir, siendo satisfactorio por cuanto la población progresivamente adopta conductas individuales que repercuten en beneficio no solo de sí mismos, sino, de la familia y sociedad ecuatoriana, debido que se cuenta con la posibilidad de elevar la expectativa y calidad de vida.

\section{REFERENCIAS CONSULTADAS}

1. Acosta, M. P. (2011). Explorando la teoría general de enfermería de Orem. Enf Neurol (Mex), 163-167. Obtenido de https://www.medigraphic.com/pdfs/enfneu/ene-2011/ene113j.pdf

2. Aldana Zavala, J., \& Isea Argüelles, J. (2019). La economía y la epidemiología social. Una praxis humana en la cosmovisión del nuevo milenio. Revista Arbitrada Interdisciplinaria de Ciencias de la Salud. Salud y Vida, 3(5), 169-181. doi:http://dx.doi.org/10.35381/s.v.v3i5.363

3. Angarita, O. M. (2014). Agencia de autocuidado en hipertensos usuarios de un hospital universitario en Cúcuta (Colombia). Salud Uninorte, 133-145. Obtenido de http://www.scielo.org.co/pdf/sun/v30n2/v30n2a05.pdf

4. Backes DS, Erdmann AL, Büscher A. (2010). O cuidado de enfermagem como práticaempreendedora: oportunidades e possibilidades. Acta Paul Enferm (3): 341-7. Disponible en: http://www.scielo.br/pdf/ape/v23n3/v23n3a05.pdf 
5. Coello, C. (29 de septiembre de 2017). Ecuador recién se interesa por las enfermedades del corazón en la mujer. Obtenido de https://www.redaccionmedica.ec/secciones/salud-publica/el-objetivo-para-estemilenio-es-controlar-la-enfermedad-cardiovascular-91029

6. Diario El Comercio. (29 de agosto de 2016). Las enfermedades cardiovasculares son la primera causa de muerte en Ecuador. Obtenido de https://www.elcomercio.com/tendencias/enfermedadescardiovascularesmuertes-ecuador-cifras-juangabriel.html

7. Naranjo Hernández, Y., Concepción Pacheco, J., \& Rodríguez Larreynaga, M. (2018). La teoría Déficit de autocuidado: Dorothea Elizabeth Orem. Gaceta Médica Espirituana, 19(3). Recuperado de http://revgmespirituana.sld.cu/index.php/gme/article/view/1129

8. Organización Mundial de la Salud. (2018). Hipertensión. Obtenido de https://www.who.int/topics/hypertension/es/

9. Rodríguez Gázquez, Ma de los Ángeles, Arredondo Holguín, Edith, \& Salamanca Acevedo, Yurany Andrea. (2013). Capacidad de agencia de autocuidado y factores relacionados con la agencia en personas con insuficiencia cardíaca de la ciudad de Medellín (Colombia). Enfermería Global, 12(30), 183-195. Recuperado de http://scielo.isciii.es/scielo.php?script=sci arttext\&pid=S1695$\underline{61412013000200009 \& \operatorname{lng}=\mathrm{es} \& \operatorname{tln} \mathrm{n}=\mathrm{es}}$.

10. Veletanga, J. (24 de junio de 2019). La prevención de enfermedades cardiovasculares se basa en un diagnóstico oportuno. Obtenido de https://www.redaccionmedica.ec/secciones/profesionales/la-prevencion-de-lasenfermedades-cardiovasculares-se-basa-en-un-diagnostico-oportuno-94335

\section{REFERENCES CONSULTED}

1. Acosta, M. P. (2011). Exploring Orem's general nursing theory. Enf Neurol (Mex), 163-167. Retrieved from https://www.medigraphic.com/pdfs/enfneu/ene2011/ene113j.pdf

2. Aldana Zavala, J., \& Isea Argüelles, J. (2019). The economy and social epidemiology. A human praxis in the worldview of the new millennium. Interdisciplinary Arbitrated Journal of Health Sciences. Health and Life, 3 (5), 169181. doi: http: //dx.doi.org/10.35381/s.v.v3i5.363 


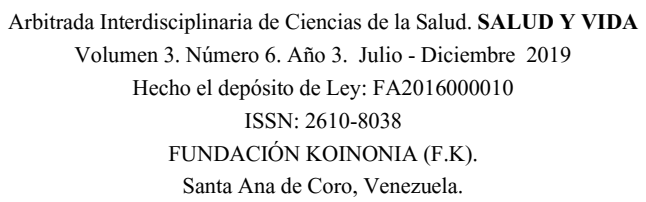

Andrés Cruz Medina; Santiago Martínez Oñate

3. Angarita, O. M. (2014). Self-care agency in hypertensive users of a university hospital in Cúcuta (Colombia). Uninorte Health, 133-145. Retrieved from http://www.scielo.org.co/pdf/sun/v30n2/v30n2a05.pdf

4. Backes DS, Erdmann AL, Büscher A. (2010). Or nursing care as an entrepreneurial practice: opportunities and possibilities. Act Paul Enferm (3): 341 7. Available at: http://www.scielo.br/pdf/ape/v23n3/v23n3a05.pdf

5. Coello, C. (September 29, 2017). Ecuador is just interested in heart disease in women. Obtained from https://www.redaccionmedica.ec/secciones/saludpublica/el-objetivo-para-este-milenio-es-controlar-la-enfermedad-cardiovascular$\underline{91029}$

6. El Comercio newspaper. (August 29, 2016). Cardiovascular diseases are the leading cause of death in Ecuador. Retrieved from https://www.elcomercio.com/tendencia/enfermedadescardiovascular-muertesecuador-cifras-juangabriel.html

7. Naranjo Hernández, Y., Concepción Pacheco, J., \& Rodríguez Larreynaga, M. (2018). The theory Self-care deficit: Dorothea Elizabeth Orem. Spiritual Medical Gazette, $\quad 19$ (3). $\quad$ Recovered from http://revgmespirituana.sld.cu/index.php/gme/article/view/1129

8. World Health Organization. (2018). Hypertension. Retrieved from https://www.who.int/topics/hypertension/es/

9. Rodríguez Gázquez, Maㅗ de los Ángeles, Arredondo Holguín, Edith, \& Salamanca Acevedo, Yurany Andrea. (2013). Self-care agency capacity and agency-related factors in people with heart failure in the city of Medellín (Colombia). Global $\begin{array}{lllll}\text { Nursing, } & 12 & \text { (30), } & \text { 183-195. } & \text { Retrieved from }\end{array}$ http://scielo.isciii.es/scielo.php?script=sci arttext\&pid=S169561412013000200009\&lng=en\&tIng=en.

10. Veletanga, J. (June 24, 2019). The prevention of cardiovascular diseases is based on a timely diagnosis. Obtained from https://www.redaccionmedica.ec/secciones/profesionales/la-prevencion-de-lasenfermedades-cardiovasulares-se-basa-en-un-diagnostico-oportuno-94335 